\title{
Opciones terapéuticas en reconstrucción después de faringolaringectomía en cáncer de laringe e hipofaringe
}

\section{Therapeutics options in reconstruction after of faringolaringectomy in larynx and hypopharyngeal cancer}

\author{
Carlos Morales A ${ }^{1}$, Mauricio Moreno V².
}

\begin{abstract}
RESUMEN
El cáncer de laringe avanzado y el cáncer de hipofaringe pueden ser tratados con terapia de conservación de órganos con radioquimioterapia, sin embargo, cuando esta última fracasa, la cirugía es la última opción para esta patología. El cáncer de hipofaringe posee una pobre sobrevida en estados avanzados, y su tratamiento quirúrgico después de radioterapia siempre se transforma en un desafío quirúrgico por las condiciones del tejido irradiado. Este artículo realiza una revisión general de las opciones de reconstrucción para defectos faringolaríngeos tanto parciales como circunferenciales, ante el caso eventual de que por la extensión tumoral y su tratamiento ablativo no sea posible un cierre primario. Se revisan distintos resultados de los principales colgajos utilizados en reconstrucción en cabeza y cuello, como son los colgajos libres fasciocutáneos, colgajo pectoral mayor y colgajos viscerales como el yeyuno, comparando tasas de fistulas faringocutáneas, resultados en deglución y rehabilitación fonatoria posterior, intentando realizar una aproximación a una recomendación terapéutica. Por último cabe mencionar que todo servicio de cabeza y cuello debe conocer las opciones existentes para cada paciente en particular, ya que además de buscar resultados oncológicos, se debe intentar lograr buenos resultados funcionales que no empobrezcan la calidad de vida de nuestros pacientes.
\end{abstract}

Palabras clave: Cáncer, laringectomía, colgajos, reconstrucción.

\begin{abstract}
Advanced laryngeal cancer and hypopharyngeal cancer can be treated with organ preservation therapy with radiochemotherapy. However, when the latter fails, surgery is the last option for this pathology. Hypopharyngeal cancer has a poor survival in advanced stages, and its surgical treatment after radiotherapy always becomes a surgical challenge due to the conditions of the irradiated tissue. This article makes a general review of the reconstruction options for both partial and circumferential pharyngolaryn-

\footnotetext{
Servicio Otorrinolaringología Hospital Barros Luco Trudeau. Universidad de Chile, Santiago, Chile.

2 Departamento de Otorrinolaringología, Cirugía Cabeza y Cuello. University of Arkansas for Medical Sciences, Estados Unidos.

*Los autores declaran no tener conflictos de interés.

Recibido el 17 de marzo, 2018. Aceptado el 19 de abril, 2018.
} 
geal defects, in the case where tumor extensions and ablative treatments do not permit a primary closure. Different results of the main flaps used in head and neck reconstruction are reviewed, such as the fasciocutaneous free flaps, pectoralis major flap and visceral flaps such as the jejunum. We then compare the rates of pharyngocutaneous fistulas, swallowing results and posterior phonation rehabilitation, while trying to perform an approach to a therapeutic recommendation. Finally, it should be mentioned that all head and neck services must know the existing options for each patient in particular, since in addition to looking for oncological results, one should try to achieve good functional results that do not impoverish the quality of life of our patients.

Key words: Cancer, laringectomy, flaps, reconstruction.

\section{INTRODUCCIÓN}

El cáncer de laringe en estadios avanzados y el cáncer de hipofaringe pueden ser tratados con la opción de conservación de órganos utilizando la combinación de radioquimioterapia ${ }^{1-4}$. Sin embargo, cuando esta opción fracasa, la cirugía de salvataje se transforma en un desafío quirúrgico por los cambios que se producen en los tejidos haciendo más complicada la resección y por ende la reconstrucción ${ }^{5}$.

Es conocido que el cáncer de hipofaringe posee un pobre pronóstico, con tasas de sobrevida libre de enfermedad de $34 \%$ y $27 \%$ en seguimientos a 3 y 5 años respectivamente ${ }^{6}$. De la misma forma se reportan mayores tasas de complicaciones como fístulas faringocutáneas y estenosis de la neofaringe con la aparición de un trastorno deglutorio secundario $0^{7-9}$. Al realizar una faringolaringectomía es de suma importancia lograr una resección completa del tumor, sin embargo, la mucosa sana para lograr un cierre primario sin presentar dificultad en la deglución posterior se ha estudiado debe ser mayor a $2,5 \mathrm{~cm}^{10}$, menor a ello es indispensable una reconstrucción del tracto aereodigestivo superior (Figura 1).

La elección de la mejor opción reconstructiva después de una cirugía primaria 0 de salvataje (laringectomía o faringectomía con resección subtotal o circunferencial) es aún un tema controversial. Desde la primera publicación por Ariyan en 197911, el colgajo pectoral mayor ha sido el standard utilizado para reconstrucción en cabeza y cuello, dado que es un colgajo bastante vital y su elevación y rotación es técnicamente simple. Por otra parte, la transposición de yeyuno también se ha utilizado para reconstrucción de defectos faringolaríngeos, sin embargo, la rehabilitación vocal es más compleja y la deglución se torna más lenta, sin mencionar la morbilidad que traduce una cirugía abdominal. Actualmente los colgajos libres son una solución técnicamente más compleja y no exenta de complicaciones o morbilidad asociada. El colgado radial fue descrito por Yang ${ }^{12}$ en 1981. Este colgajo entrega una buena superficie cutánea

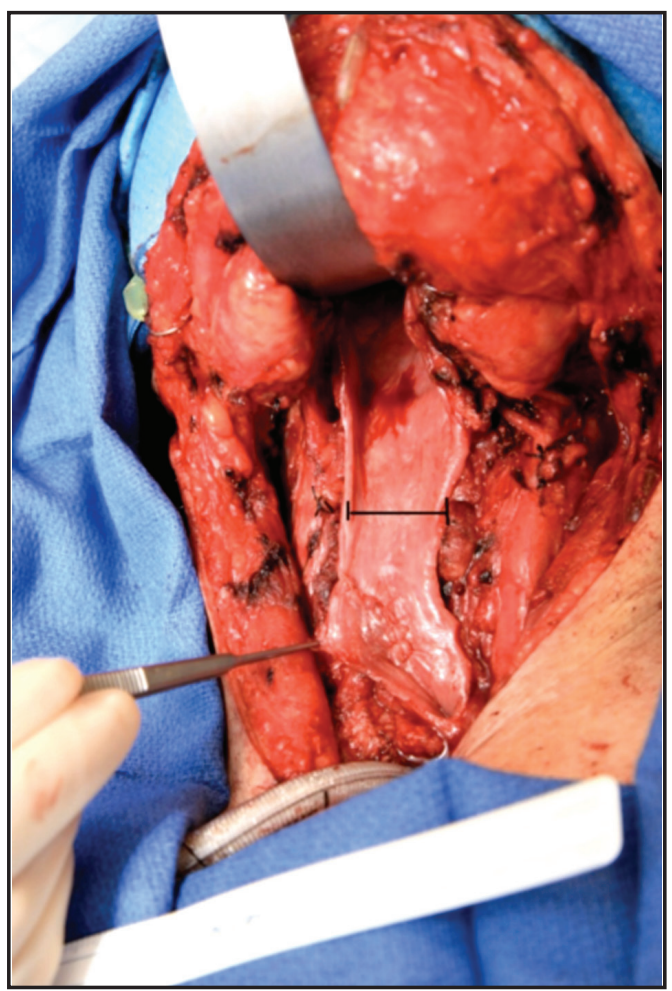

Figura 1. Medida de mucosa faríngea posterior necesaria para cierre primario. Menos de 2,5 cm es indicación de reconstrucción. 
que puede fácilmente reparar el defecto dado por la resección tumoral, y además posee una longitud adecuada para la anastomosis vascular con el cueIlo ipsilateral o contralateral al defecto. De la misma forma dentro de las opciones de colgajos libres se encuentra el colgajo anterolateral de muslo, el cual ha tomado popularidad en el último tiempo dado que provee una superficie cutánea excelente con un largo pedículo vascular, además que al estar lejos del sitio del defecto permite trabajar con otro equipo quirúrgico que realice la etapa resectiva y otro que levante el colgajo.

El propósito de este artículo es revisar las distintas opciones de reconstrucción para un defecto faringolaríngeo, sus ventajas y desventajas, además de entregar un algoritmo que permita orientar en la toma de decisión ante un eventual desafío quirúrgico.

\section{Opciones quirúrgicas}

\section{- Colgajo pectoral mayor}

La técnica actualmente utilizada es la descrita por Spriano ${ }^{13}$, con tasas de fístulas faringocutáneas que van de $0 \%$ a $57 \%$ con una media de $27 \%$, y tasas de estenosis distal que van de $0 \%$ a $43 \%$ con una media de $17 \%$ según lo descrito en distintas series ${ }^{14-16}$. Una de las más importantes ventajas del colgajo pectoral es la menor complejidad que presenta levantar el colgajo, con un tiempo quirúrgico menor comparado con otras opciones. Además el componente muscular del colgajo puede utilizarse para cubrir los grandes vasos del cuello después de una disección cervical radical de cuello y prevenir así un síndrome de estallido carotídeo, especialmente en pacientes intervenidos posteriormente a la radioquimioterapia (Figura 2).

El entrenamiento de reconstrucción microvascular no es necesario y por ende puede ser realizado en un centro con bajo volumen de pacientes oncológicos, aunque con un servicio de cabeza y cuello familiarizado con este tipo de pacientes. Es interesante que distintas publicaciones han identificado que el uso de colgajo pectoral en reconstrucción de defectos hipofaríngeos es un factor de riesgo independiente para la falla de la reconstrucción y uso permanente de gastrostomía. Esto principalmente a que la dehiscencia y necrosis del colgajo se traduce en una fístula faringocutánea, que en tejidos irradiados se transforma en un defecto crónico, prolongando la estadía hospitalaria, aumentando la morbilidad y los costos asociados.

Por lo tanto, se debe considerar que esta opción no debiera utilizarse si el defecto faringolaríngeo es mayor de 11-12 cm, si se resecó parte importante de la base de lengua 0 de las paredes laterales de la orofaringe, y esto porque el colgajo que se levanta es voluminoso y se produce un

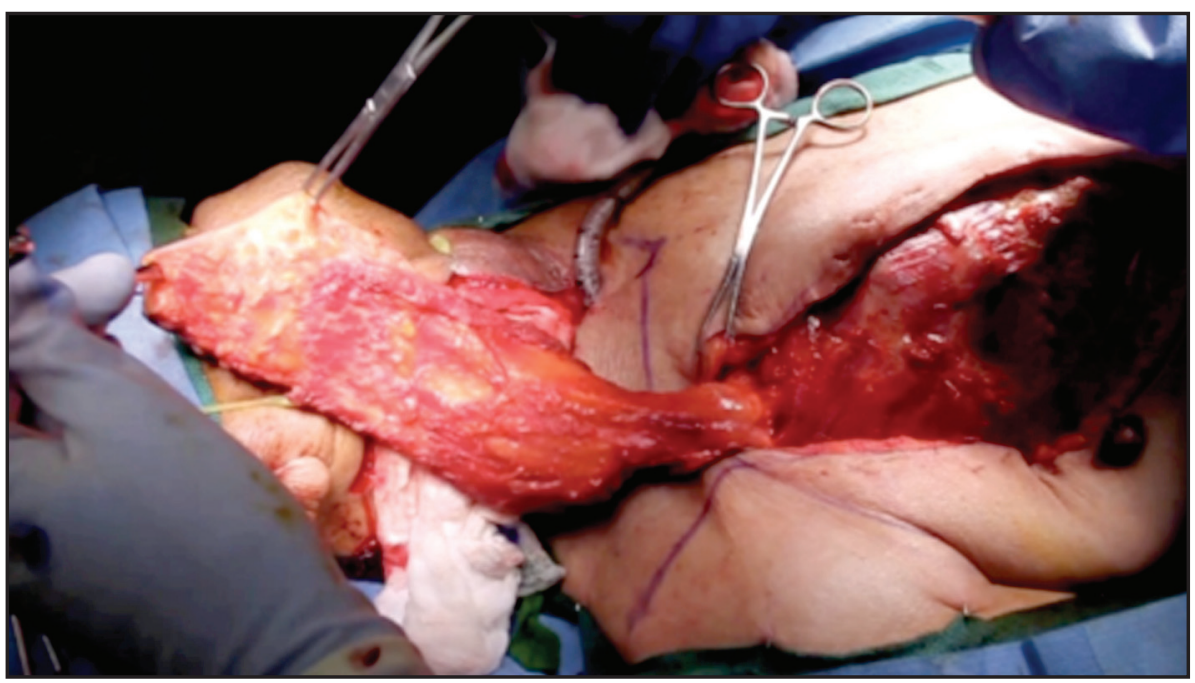

Figura 2. Colgajo pectoral mayor con su pedículo de la arteria toracoacromial. 
fenómeno de tracción sobre el defecto. En conclusión, el colgajo pectoral debiese ser considerado como segunda opción, limitado a defectos faringolaríngeos parciales, o en pacientes con un estado nutricional 0 con presencia de morbilidades que impidan una cirugía mayor como sería una reconstrucción con colgajos libres.

\section{- Colgajos viscerales}

El colgajo de yeyuno es la técnica más antigua utilizada para reconstrucciones de defectos circunferenciales hipofaríngeos y de esófago cervical. Fue descrito en 1959 por Seidenberg ${ }^{17}$, y rápidamente fue utilizado por los cirujanos de la época. El concepto de un injerto tubular que pudiese ser anastomosado al defecto resultante de la resección fue muy interesante, sin embargo, las tasas de fístula con una media de $12 \%$ y estenosis distal de $11 \%$ son absolutamente comparables con los obtenidos con menos complejidad y menos morbilidad por los colgajos libres fasciocutáneos. De hecho, la mortalidad perioperativa de un colgajo de yeyuno varía entre $0 \%$ a $17 \%$, con una media de $2,5 \%$, lo cual es cuatro veces mayor a lo reportado para un colgajo microvascular fasciocutáne $0^{17-19}$. Por otro lado, una de las mayores desventajas es la limitada tolerancia a la isquemia, con un tiempo menor para la anastomosis microvascular, con potenciales alteraciones tisulares que predispongan a la pérdida del colgajo.

\section{- Colgajos libres fasciocutáneos}

Dentro de esta variedad son varias opciones que se disponen para realizar una transferencia tisular exitosa, los más utilizados en la actualidad son los colgajos radiales y el anterolateral de muslo. El colgajo radial permite realizar una reconstrucción exitosa de defectos faringolaríngeos parciales y circunferenciales, la tasa de fístula faringocutánea varía entre $2 \%$ y $53 \%$ con una media de $20 \%$, y la de estenosis del tubo digestivo varía en tasas entre 0\%-36\% con una media de $11 \%$ según lo reportado en distintas series ${ }^{20-23}$. Resultados levemente mejores que los observados para el colgajo pectoral, y con similares cifras a colgajos viscerales pero con menos morbilidad. Sin embargo, se ha reportado que las fístulas faringocutáneas cierran en mayor porcentaje de forma espontánea en colgajos fasciocutáneos libres que en otro tipo de reparación, sin requerir manejos complejos de ésta. En el colgajo radial se puede obtener una paleta cutánea hasta de 12-14 $\mathrm{cm}$ sin mayor dificultad, incluso llegar a los $18 \mathrm{~cm}$ en situaciones críticas, lo cual es suficiente para el manejo de la mayoría de los defectos hipofaríngeos y esofágicos cervicales (Figura 3).

La buena flexibilidad y grosor de este colgajo es una excelente opción en pacientes obesos, en los cuales, el colgajo pectoral y el anterolateral de muslo están contraindicados por su gran volumen. Además se obtienen mejores resultados

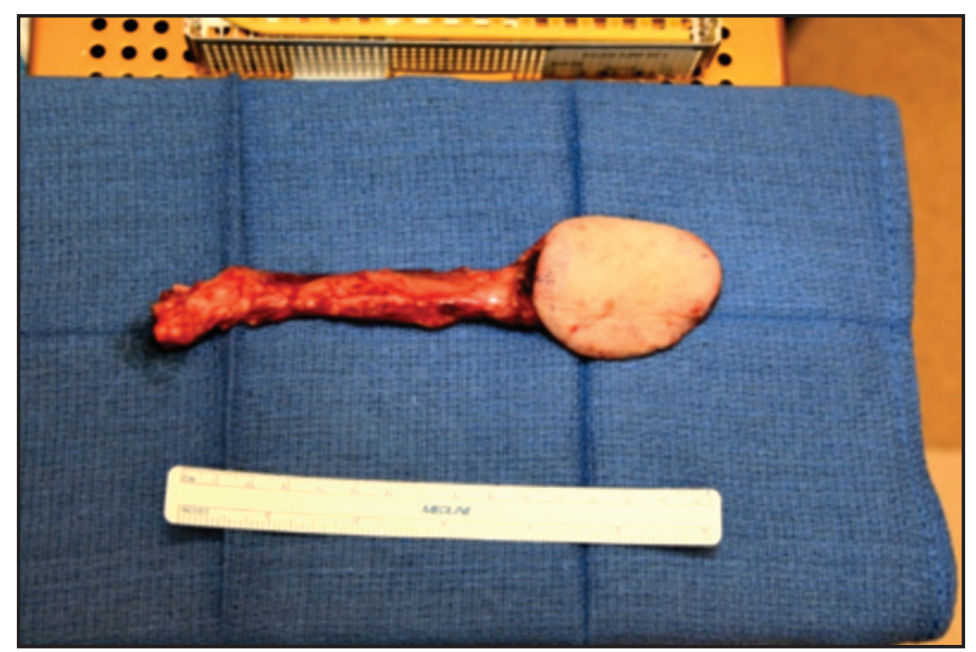

Figura 3. Colgajo radial con su paleta cutánea. 
funcionales del punto de vista de la deglución y rehabilitación fonatoria, dado lo delgado del colgajo y su similitud con la mucosa faríngea. El colgajo radial no está exento de complicaciones a corto 0 largo plazo, algunas de ellas son la disminución del tiempo de cicatrización del sitio donante, exposición de los tendones del antebrazo, rigidez 0 resultados estéticos desfavorables. Sin embargo esto no se traduce en disminución de la función articular ni mayores impactos en la calidad de vida de los pacientes ${ }^{24}$.

El colgajo anterolateral de muslo fue descrito por Song en $1984^{25}$, y rápidamente comenzó a ser utilizado por los cirujanos dedicados a la reconstrucción en cabeza y cuello. Sus resultados han sido reportados en cifras que van entre 0\%-25\% con una media de $15 \%$ para fístula faringocutánea y $0 \%-30 \%$ con una media de $9 \%$ para estenosis de la neofaringe ${ }^{26-28}$. La tasa de fístula es evidentemente menor, lo que puede ser explicado por el uso de fascia lata como primera capa de cierre y una robusta segunda capa compuesta por el resto del colgajo. La extensión de la paleta cutánea es mayor que el colgajo radial, por lo que puede ser utilizado sin dificultades para cierre de defectos circunferenciales faringolaríngeos o también en casos de defectos cutáneos cervicales, se pueden utilizar 2 paletas cutáneas para la reconstrucción de la neofaringe y del defecto en piel si es necesario, los cuales son conocidos como colgajos quiméricos (Figura 4).
La tasa de mortalidad relacionada con este flap es prácticamente inexistente y la morbilidad del sitio donante es la más baja de los colgajos libres, cabe recordar que el defecto del sitio donante se puede cerrar de forma primaria sin mayor dificultad. Los resultados estéticos son totalmente aceptables, sin existir mayores problemas para caminar posteriormente, con una rápida recuperación. La deglución después de un colgajo anterolateral de muslo obtiene los mejores resultados comparándolos con otros colgajos libres ${ }^{29}$. La principal contraindicación de este colgajo es la enfermedad vascular periférica, por lo cual es indispensable un estudio angiográfico previo a la cirugía. Traumas previos de la zona y obesidad pueden ser consideradas contraindicaciones relativas, en estos casos la opción del colgajo radial será la más adecuada.

\section{DISCUSIÓN}

Distintas publicaciones disponibles en la literatura ${ }^{29,30}$, reportan altas tasas de incidencia de complicaciones menores y dehiscencia en reconstrucciones utilizando colgajo pectoral mayor, sin embargo, hay que recordar que éstos son utilizados por su menor tiempo quirúrgico y complejidad técnica lo que beneficia a pacientes con una pobre condición de salud con múltiples morbilidades.

La evaluación funcional presenta diferencias importantes entre el uso de colgajos fasciocutá-

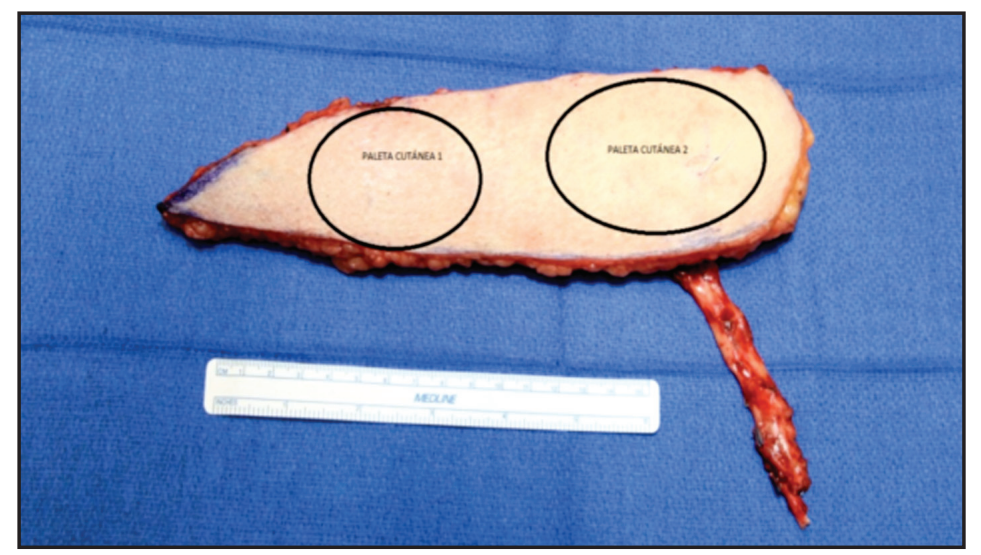

Figura 4. Colgajo anterolateral de muslo. Se pueden obtener dos paletas cutáneas, también llamados colgajos quiméricos. 
neos libres y los colgajos viscerales o regionales como el pectoral mayor. La dificultad para lograr una adecuada deglución causa un importante estrés psicológico en estos pacientes, por lo tanto un resultado exitoso en este aspecto resulta de suma importancia. Solo $5 \%$ de los pacientes en los cuales se utilizó colgajos libres fasciocutáneos necesitó de gastrostomía para alimentarse, y 94\% de ellos logró una alimentación oral. Una alimentación con una dieta completamente normal fue reportada en tasas del $75 \%$ para este tipo de colgajos comparados con solo $59 \%$ para los colgajos viscerales ${ }^{31}$.

Por otro lado, al comparar las fístulas faringocutáneas no existe una diferencia significativa entre una opción y otra, aun cuando ésta es menor en el caso de colgajos libres fasciocutáneos especialmente el anterolateral de muslo, sin embargo, sí existe una diferencia significativa en el tiempo de cierre cuando se comparan los fasciocutáneos con los viscerales y regionales, los primeros en promedio tardan entre 14 a 19 días en cerrar con manejo espontáneo, mientras que el resto demora entre 3 a 20 semanas, lo cual es significativamente perjudicial cuando hablamos de mayores costos hospitalarios, días/cama, y el retraso al iniciar un eventual tratamiento complementario como es la radioquimioterapia. No existen diferencias en la terapia de cierre, la mayoría de las opciones elegi- das logra un cierre en alrededor del $65 \%$ solo con manejo expectante ${ }^{32}$. La tasa de fracaso del colgajo fue reportada en orden del $2 \%-5 \%$ para los colgajos fasciocutáneos, siendo significativamente menor al compararlos con los viscerales. Los costos relacionados con días/cama, uso de unidades de cuidados intensivos, uso de ventilador mecánico fue menor para el uso de colgajo anterolateral de muslo al compararlo con colgajos entéricos, aunque similar con el uso de colgajo pectoral.

Algunos autores sugieren que la reconstrucción con colgajos fasciocutáneos tiene como ventaja fundamental la menor morbilidad del sitio donante. Un ejemplo de ello es que el colgajo de yeyuno presenta $5,8 \%$ de complicaciones abdominales, las que pueden incluir: dehiscencia de la pared, obstrucción intestinal, hemorragia, ileo prolongado, hematoma, entre otras ${ }^{33}$. El colgajo anterolateral de muslo cicatriza rápidamente, el paciente puede deambular al segundo día poscirugía y llevar una vida normal en un plazo promedio de 10 días. Clark ${ }^{14}$, postuló un algoritmo que puede ser utilizado como guía para reconstrucción de defectos faringolaríngeos, siempre considerando a cada paciente como un caso particular el cual debe ser evaluado según su condición clínica, además de la experiencia y recursos disponibles en cada centro (Figura 5).

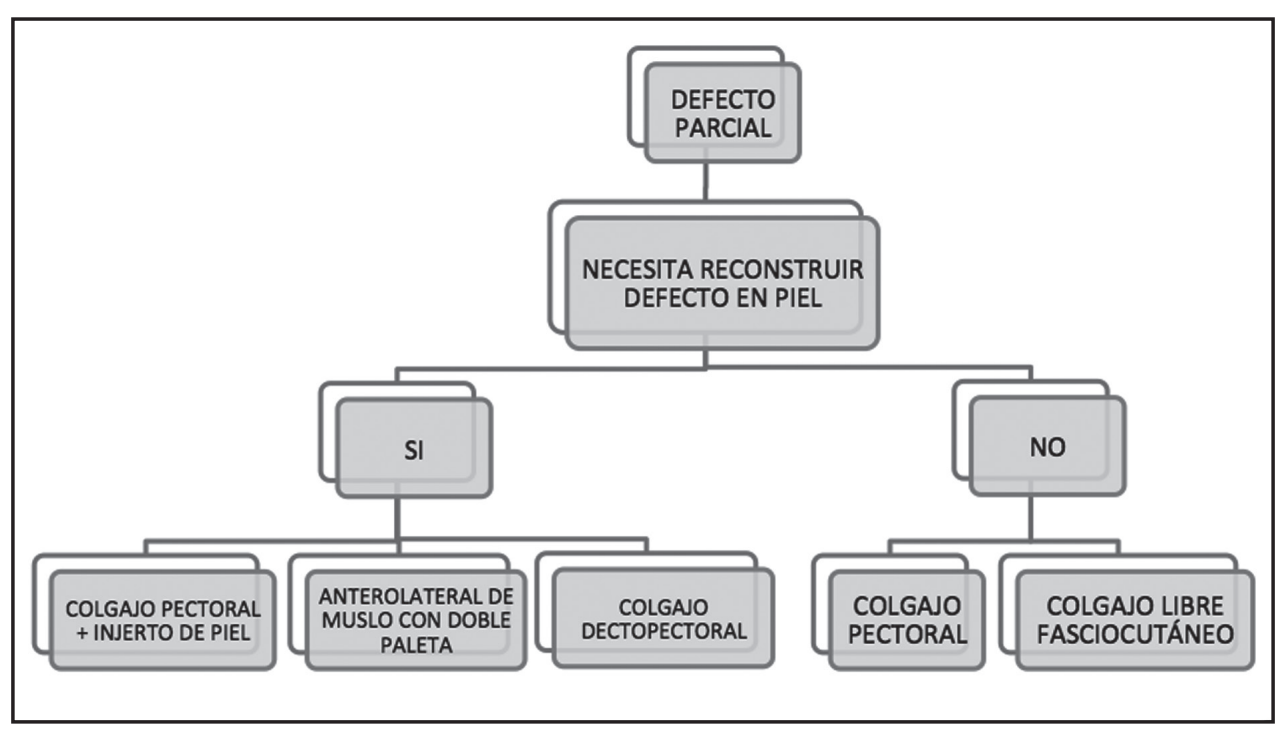

Figura 5. Algoritmo de reconstrucción para defectos faringolaríngeos parciales. 


\section{CONCLUSIÓN}

Una sola técnica no puede ser utilizada como regla en reconstrucción de defectos faringolaríngeos, debido a los distintos desafíos que presenta un caso en particular. Sin embargo, los otorrinolaringólogos y cirujanos de cabeza y cuello deben estar al conocimiento y poseer un amplio campo de opciones para elegir la más adecuada ante un eventual escenario que requiera reconstrucción. Esto es crítico

\section{BIBLIOGRAFÍA}

1. Forastiere aA, Goepfert h, Maor M, et al. Concurrent chemotherapy and radiotherapy for organ preservation in advanced laryngeal cancer. N Engl J Med 2003; 349: 2091-8.

2. Wolf GT, Hong WK. Induction chemotherapy for organ preservation in advanced laryngeal cancer: is there a role? Head Neck 1995; 17: 279-83.

3. Lefebvre JL, Chevalier D, Luboinski B, et al. Larynx preservation in pyriform sinus cancer: preliminary results of a European Organization for Research and Treatment of Cancer phase III trial. EORTC Head and Neck Cancer Cooperative Group. J Natl Cancer Inst 1996; 88: 890-9.

4. Lefebvre Jl, Rolland F, Tesselaar M, et al. Phase 3 randomized trial on larynx preservation comparing sequential vs alternating chemotherapy and radiotherapy. J Natl Cancer Inst 2009; 101: 142-52.

5. Van der Putten L, De Bree L, Doornaert P, Buter J, Eerenstein E, Kuik D, Leemans C. Salvage surgery in post-chemoradiation laryngeal and hypopharyngeal carcinoma: outcome and review. Acta otorhinolaryngologica italica 2015; 35: 16272.

6. Donnadieu J, Klopp-Dutote D, Biet-Hornstein A, Strunski V, Mortuaire G, Page C. Therapeutic Management of Pyriform Sinus Cancer. Otolaryngol Head Neck Surg 2017; 156: 498503.

7. Stoeckli SJ, Pawlik AB, Lipp M, et al. Salvage surgery after failure of nonsurgical therapy for carcinoma of the larynx and hypopharynx. Arch Otolaryngol Head Neck Surg 2000; 126: 1473-7.

8. Tsou YA, Hua CH, LIN, et al. Comparison of pharyngocutaneous fistula between patients cuando se presentan casos con tumores extensos en los cuales se sabe de antemano que el defecto puede llegar a ser circunferencial, extendiéndose a orofaringe 0 esófago cervical, o en el caso de cirugías de salvataje después de protocolos de conservación de órganos fallidos. Siempre es bueno recordar que además de realizar una adecuada cirugía ablativa, se debe pensar en la etapa de reconstrucción para así evitar disminuir la funcionalidad e independencia de estos pacientes que se traduce en calidad de vida.

followed by primary laryngopharyngectomy and salvage laryngopharyngectomy for advanced hypopharyngeal cancer. Head Neck 2010; 32: 1494-500.

9. Patel UA, Moore BA, Wax M, et al. Impact of pharyngeal closure technique on fistula after salvage laryngectomy. JAMA Otolaryngol Head Neck Surg 2013; 139: 1156-62.

10. Y. HuI, W.I. WeI, P.W. Yuen. Primary Closure of Pharyngeal Remnant After Total Laryngectomy and Partial Pharyngectomy: How Much Residual Mucosa Is Sufficient? Laryngoscope 1996; 106: 490-4.

11. ARIYIAN S. The pectoralis major myocutaneous flap: a versatile flap for reconstruction in the head and neck. Plast Reconstr Surg 1979; 63: 73-81.

12. Yang G, Chen B, Gao Y, et al. Forearm free skin flap transplantation. Natl Med J China 1981; 61: 139.

13. Spriano G, Piantanida R, Pellini R. Hypopharyngeal reconstruction using pectoralis major myocutaneous flap and prevertebral fascia. Laryngoscope 2001; 111: 544-7.

14. Clark JR, GILbert R, Irish J, et al. Morbidity after flap reconstruction of hypopharyngeal defects. Laryngoscope 2006; 116: 173-81.

15. Jegoux F, Ferron C, Malard 0, et al. Reconstruction of circumferential pharyngolaryngectomy using a 'horseshoe-shaped' pectoralis major myocutaneous flap. J Laryngol Otol 2007; 121 : 483-8.

16. Cristalli G, Pellini R, Roselli R, et al. Pectoralis major myocutaneous flap for hypopharyngeal reconstruction: long-term results. J Craniofac Surg 2011; 22: 581-4.

17. Lewin JS, Barringer DA, May AH, et al. Functional outcomes after circumferential 
pharyngoesophageal reconstruction. Laryngoscope 2005; 115: 1266-71.

18. $Y \cup P$, Roв GL. Pharyngoesophageal reconstruction with the anterolateral thigh flap: a clinical and functional outcomes study. Plast Reconstr Surg 2005; 116: 1845-55.

19. Lewin JS, Barringer DA, May AH, et al. Functional outcomes after laryngopharyngectomy with anterolateral thigh flap reconstruction. Head Neck 2006; 28: 142-9.

20. Azizzadeh B, Yafal S, Rawnsley JD, et al. Radial forearm free flap pharyngoesophageal reconstruction. Laryngoscope 2001; 111: 80710.

21. Scharpf J, Esclamado RM. Reconstruction with radial forearm flaps after ablative surgery for hypopharyngeal cancer. Head Neck 2003; 25: 261-6.

22. Andrades P, Pehler SF, Baranano CF, et al. Fistula analysis after radial forearm free flap reconstruction of hypopharyngeal defects. Laryngoscope 2008; 118: 1157-63.

23. Yang CC, Lee JC, Wu KC, et al. Voice and speech outcomes with radial forearm free flap-accompanied phonation tube after total pharyngolaryngectomy of hypopharyngeal cancer. Acta Otolaryngol 2011; 131: 847-51.

24. Song YG, Chen GZ, Song YL. The free thigh flap: a new free flap concept based on the septocutaneous artery. Br J Plast Surg 1984; 37: 149-59.

25. Swanson E, Boyd JB, Manktelow RT. The radial forearm flap: reconstructive applications and donor-site defects in 35 consecutive patients. Plast Reconstr Surg 1990; 85: 258-66.

26. Murray DJ,Gilbert RW, Vesely MJJ, et al. Functional outcomes and donor site morbidity following circumferential pharyngoesophageal reconstruction using an anterolateral thigh flap and salivary bypass tube. Head Neck 2007; 29: 147-54.

27. Sagar B, Marres haM, Hartman eHM. Hypopharyngeal reconstruction with an anterolateral thigh flap after laryngopharyngeal resection: results of a retrospective study on 20 patients. J Plast Reconstr Aesthet Surg 2010; 63: 970-5.

28. Yu P, Hanasono MM, Skoracki RJ, et al. Pharyngoesophageal reconstruction with the anterolateral thigh flap after total laryngopharyngectomy. Cancer 2010; 116: 1718-24.

29. Murray DJ, Novak CB, Neligan PC. Fasciocutaneous free flaps in pharyngolaryngo-oesophageal reconstruction: a critical review of the literature. J Plast Reconstr Aesthet Surg 2008; 61: 1148-56.

30. Taeyul Lee, Chulhoon Chung, Yonguoon Chang, Jaehyun Kim. Comparison of Clinical and Functional Outcomes Using Pectoralis Major and Cutaneous Free Flaps for Hypopharyngeal Squamous Cell Carcinoma. Arch Plast Surg 2015; 42: 608-13.

31. Reece GP, Schusterman Ma, Miller MJ, et al. Morbidity and functional outcome of free jejunal transfer reconstruction for circumferential defects of the pharynx and cervical esophagus. Plast Reconstr Surg 1995; 96: 1307-16.

32. Yu P, Lewin JS, Reece GP, et al. Comparison of clinical and functional outcomes and hospital costs following pharyngoesophageal reconstruction with the anterolateral thigh free flap versus the jejunal flap. Plast Reconstr Surg 2006; 117: 968-74.

33. Shangold LM, URken ML, Lawson W. Jejunal transplantation for pharyngoesophageal reconstruction. Otolaryngol Clin North Am 1991; 24: 1321-42.

Correspondencia: Carlos Morales A.

Hospital Barros Luco Trudeau. Santiago, Chile

E mail:dr.cmorales.a@gmail.com 